\title{
Short hairpin RNA targeting autotaxin reduces human gastric carcinoma AGS cell proliferative, migratory and invasive capabilities in vitro and causes tumor regression in vivo
}

\author{
ZHOUXUN CHEN $^{1}$, YINGPENG HUANG ${ }^{1}$, XIAN SHEN $^{1}$, JIAN GUO ${ }^{1}$, \\ GUANBAO ZHU $^{1}$, HENNING DRALLE ${ }^{2}$ and CUONG HOANG-VU ${ }^{2}$
}

\author{
${ }^{1}$ Department of General Surgery, The First Affiliated Hospital of Wenzhou Medical School, Wenzhou, \\ Zhejiang 325000, P.R. China; ${ }^{2}$ Research Group of Experimental and Surgical Oncology, Department of General, \\ Visceral and Vascular Surgery, Martin Luther University Halle-Wittenberg, D-06097 Halle, Germany
}

Received September 25, 2012; Accepted November 5, 2012

DOI: $10.3892 / o r .2012 .2204$

\begin{abstract}
The aim of this study was to investigate the effect of short hairpin RNA (shRNA) targeting autotaxin (ATX) on the migratory and invasive capability of AGS human gastric cancer cells and the growth of xenografts in nude mice. pSUPER-ATX and pSUPER-mock (non-specific), which were constructed corresponding to the ATX-shRNA and negative control mock-shRNA synthesized based on gene sequence, were transfected with blank plasmid pSUPER-control into AGS human gastric cancer cells using Lipofectamine. At 24, 48 and $72 \mathrm{~h}$ post-transfection, the cells were harvested and analyzed. The endogenous ATX mRNA and protein of the different groups of AGS cells were detected by RT-PCR and western blot assays. Cell proliferation was measured by MTT assay. In vitro Transwell and Matrigel assays were used to detect the cell migratory and invasive capabilities. A tumor xenograft model was generated by subcutaneous injection of AGS cells into the dorsum of nude mice. The growth of xenograft tumors was monitored and measured; changes in tumor morphology and the organs of mice were observed by H\&E staining. The expression of ATX, MMP-2 and MMP-9 protein in xenograft tumor tissues was detected by immunohistochemistry and western blotting. In vitro, both the mRNA and protein levels of ATX in the pSUPER-ATX group were significantly downregulated $(\mathrm{P}<0.01)$, and the cell proliferative, migratory and invasive capabilities were also significantly decreased. In vivo, no obvious damage to the organs was found. pSUPER-ATX significantly suppressed the tumor volume and weight from the 7th week after cell transplantation, compared to the pSUPER-
\end{abstract}

Correspondence to: Dr Zhouxun Chen, Department of General Surgery, The First Affiliated Hospital of Wenzhou Medical School, Fuxue Road 2, Wenzhou, Zhejiang 325000, P.R. China

E-mail: zhouxun.chen@googlemail.com

Key words: RNA interference, shRNA, gastric carcinoma, autotaxin, nude mice, xenograft mock, pSUPER-control and WT groups; the inhibition rates were approximately $50 \%(\mathrm{P}<0.05)$. However, no significant differences in these parameters were found among the WT, pSUPER-mock and pSUPER-control groups. Furthermore, ATX, MMP-2 and MMP-9 were expressed at significantly lower levels in the pSUPER-ATX group compared to the levels in the other three groups $(\mathrm{P}<0.05)$, and a significant correlation between ATX and MMP-2 expression was found $(r=0.869$, $\mathrm{P}<0.01)$. The specific shRNA targeting ATX downregulated the endogenous ATX of AGS human gastric cancer cells, and inhibited AGS cell proliferative, migratory and invasive potentials. Moreover, shRNA targeting ATX inhibited the growth of human gastric cancer xenografts in nude mice.

\section{Introduction}

Autotaxin is an extracellular phosphodiesterase and a cell autocrine motility-stimulating factor initially isolated from human malignant melanoma A2058 cell medium and purified by M.L. Stracke. Autotaxin (ATX) is a $125-\mathrm{kDa}$ glycoprotein reported to stimulate both random or directed motility of melanoma cells (1).

Protein sequence analysis indicates that there exists a significant homology between ATX and hemolytic phospholipase D on the cell surface (2). Hydrolysis of lysophosphatidylcholine by ATX in lysophosphatidic acid occurs when ATX is released into the extracellular space with deletion of amino acid $(2,3)$. Recently, ATX was found to be closely linked to angiogenesis in embryo development. In previous studies, ATX expression was investigated in thyroid carcinoma (4), malignant melanoma (5), breast carcinoma (6-8), non-small cell lung carcinoma (9), renal cell carcinoma (10) and neuroblastoma (11), while the role of ATX in hepatic carcinoma (12) has been documented in China. Matrix metalloproteinases MMP2 and MMP3, reported to be closely associated with gastric cancer infiltration and lymphatic metastasis, are used as molecular markers for detecting and evaluating the prognosis of gastric cancer $(13,14)$.

In our in vitro study, the plasmid vectors containing shRNA fragments targeting ATX were transfected into the human 
gastric cancer cell line AGS, to prove that shRNA effectively and consistently inhibits ATX mRNA and protein expression in AGS cells and reduces their abilities for proliferation, migration and invasion. Our study also compared and observed in vivo the inhibitory effect of shRNA on the growth of human gastric cancer xenografts in nude mice. These observations indicate the relationship between ATX and gastric cancer infiltration and metastasis, thereby providing the laboratory data for clarifying the molecular mechanisms of gastric cancer development and treatment.

\section{Materials and methods}

Human cell lines and culture. The human gastric cancer cell line AGC was cultured in Dulbecco's modified Eagle's medium (DMEM), supplemented with $10 \%$ foetal bovine serum (Invitrogen, Carlsbad, CA, USA) and incubated at $37^{\circ} \mathrm{C}$ in $5 \% \mathrm{CO}_{2}$.

RNA extraction, cDNA synthesis and RT-PCR. Total RNA was extracted from cell pellets by TRIzol (Invitrogen) and reverse transcribed into cDNA using Reverse Transcriptase (Applied Biosystems, Foster City, CA, USA) according to the manufacturer's instructions. The target gene expression was determined by RT-PCR using specific primers of target genes (5'-GTT GCA AGG AAA CCT TTG GA-3'; 5'-AAC TTC CTC TGG CAT GGT TG-3'). GAPDH served as an internal control for total cDNA content. Samples were amplified using the ABI Prism 7700 Sequence Detection system (Applied Biosystems).

Cell proliferation assay. The cells were cultured in well plates $\left(\sim 2 \times 10^{3} /\right.$ well). After culture for $24,48,72$ and $96 \mathrm{~h}, 20 \mu 1$ of MTT solution was added to each well. After another $4 \mathrm{~h}$ of culture, the supernatant was discarded and $150 \mu \mathrm{l}$ of DMSO was added to each well while shaking at room temperature for $10 \mathrm{~min}$, so that the crystals could be completely dissolved. The assessment procedure was repeated 3 times and the average value was determined from each well as the absorbance value at $490 \mathrm{~nm}$ on a microplate reader.

Cell migration and invasion assays. In this assay, an $8-\mu \mathrm{m}$ pore-sized polycarbonate membrane Transwell chamber (24-well plate) purchased from Corning was used. After incubation for $1 \mathrm{~h}$ at $37^{\circ} \mathrm{C}$ in a basolateral chamber containing $600 \mu 1$ RPMI-1640 medium with $20 \%$ FCS, the different gastric cancer cells were washed with PBS for 3 times. After trypsin digestion, the cells were resuspended into RPMI-1640 medium with $10 \%$ FBS. Subsequently, a 100- $\mu 1$ cell suspension $\left(1 \times 10^{6} \%\right.$ $\mathrm{ml}$ cells) was added to each well of the apical chamber and incubated in $5 \% \mathrm{CO}_{2}$ at $37^{\circ} \mathrm{C}$. The cells which were unable to pass through the microporous membrane were wiped from the apical chamber with cotton swabs. Following a further wash in PBS and fixation with $4 \%$ formalin, cells on the surface of the microporous membrane were counted randomly in five x 200 microscopic fields using Giemsa staining. This procedure was repeated 3 times for each sample, and the cell migratory ability was determined based on the number of migrating cells (based on the average value in the assay). In the cell invasion assay, as distinguished from the cell migration assay, the extracellular matrix Matrigel (BD Pharmingen, San Diego, CA, USA) was additionally used to simulate the in vivo extracellular matrix environment. Matrigel at $50 \mu \mathrm{l} / \mathrm{cm}^{2}$ was added to the surface of the polycarbonate membrane in the Transwell chamber, and then placed under a fume hood either at $37^{\circ} \mathrm{C}$ or at room temperature for $30 \mathrm{~min}$ until the Matrigel gelled. The procedure was then performed identical to the migration assay. The cell invasive ability was determined based on the number of invading cells.

Establishment of the tumor xenograft model. Twenty mice were randomly divided into 4 groups $(\mathrm{n}=5)$, inoculated with the respective cells: wild-type (WT) group, transfection targeted interference vector pSUPER-ATX group, negative control group pSUPER-mock and blank plasmid group pSUPERcontrol. The mice were subcutaneously inoculated in the right hind lateral leg with the cells $\left(2 \times 10^{7} / \mathrm{ml}\right)$ in logarithmic growth period. Then every 3 days after tumorigenesis in the nude mice, the measurements of the tumor diameter (a) and short diameter (b) with a vernier caliper were used to calculate the tumor volume according to the formula $\left(\mathrm{ax} \mathrm{b}^{2} / 2\right)$, and the tumor growth curves were drawn on the basis of the average values of the tumor volume from each group. In the 8th week when the mice were sacrificed, the tumors were extracted in order to measure the final volume and weight by using a photoelectric balance. The tumor growth inhibition rate was determined using the volume and weight. The tumor tissue of each group was stored in liquid nitrogen. Part of the tissue was fixed with $10 \%$ formaldehyde solution in order to be used for H\&E staining, immunohistochemistry and western blot experiments.

Statistical methods. Data are presented as the means \pm SD. Analysis of the significance of differences between 2 groups was performed by the two tailed Student's t-test and one-way ANOVA analysis. $\mathrm{P}<0.05$ was considered to indicate a statistically significant result.

\section{Results}

Differential ATX expression in AGS WT and transfected cells. RT-PCR was used to detect changes in ATX mRNA expression and showed that ATX mRNA expression in the transfected cells in the two cloned cell groups pSUPER-ATX1 and PSUPER-ATX2 decreased $79.34 \pm 9.95$ and $95.42 \pm 1.93 \%$, respectively, in comparison with WT cells and expression levels were was also markedly inhibited by shRNA $(t=7.247$, $\mathrm{P}<0.01 ; \mathrm{t}=14.391, \mathrm{P}<0.01$; Fig. 1A), while there was no significant difference in ATX mRNA expression among the WT, pSUPER-control and pSUPER-mock groups.

Western blot analysis was used to detect changes in ATX protein expression and showed that the ATX protein expression of the transfected cells in the two cloned cell groups pSUPER-ATX1 and pSUPER-ATX2 decreased 78.45 \pm 5.45 and $85.42 \pm 3.56 \%$, respectively, in comparison with the WT cells. ATX protein expression levels were also markedly inhibited by shRNA ( $\mathrm{t}=8.457, \mathrm{P}<0.01$; $\mathrm{t}=11.936, \mathrm{P}<0.01$; Fig. $1 \mathrm{~B})$, while there was no significant difference in ATX protein expression between the WT and negative control groups. pSUPER-ATX2 was found to be superior to pSUPER-ATX1 
A
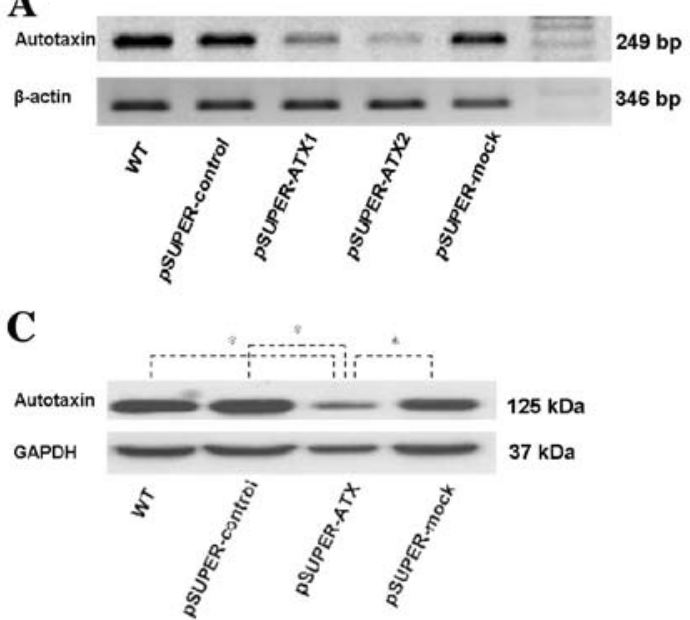

B

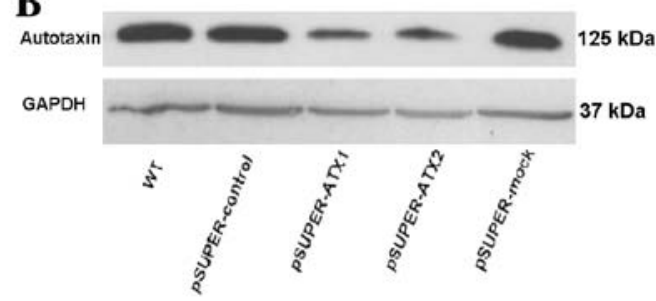

D

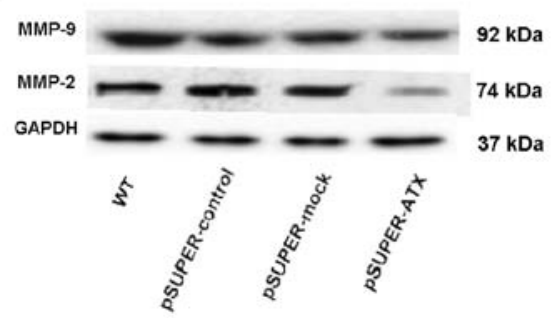

Figure 1. (A) Changes in ATX mRNA expression after transfection in the WT AGS group, pSUPER-control group, pSUPER-ATX groups (pSUPER-ATX1 and pSUPER-ATX2 as two cloned groups) and the pSUPER-mock group. (B) Changes in ATX protein expression after transfection in the WT AGS group, pSUPER-control group, pSUPER-ATX groups (pSUPER-ATX1 and pSUPER-ATX2 as two cloned groups) and the pSUPER-mock group. (C) ATX protein expression in each xenograft tumor group (western blotting). Comparison between ATX protein expression in the xenografted nude mouse tumors from the recombinant plasmid transfection group and other groups ("P<0.01). (D) Protein expression of MMP-9, MMP-2 and GAPDH in each group of xenograft tumors (western blotting). Compared with other groups, protein expression of MMP-9 and MMP-2 in transplanted tumors established by pSUPER-ATX was noticeably reduced. The difference was significant $(\mathrm{P}<0.05)$.
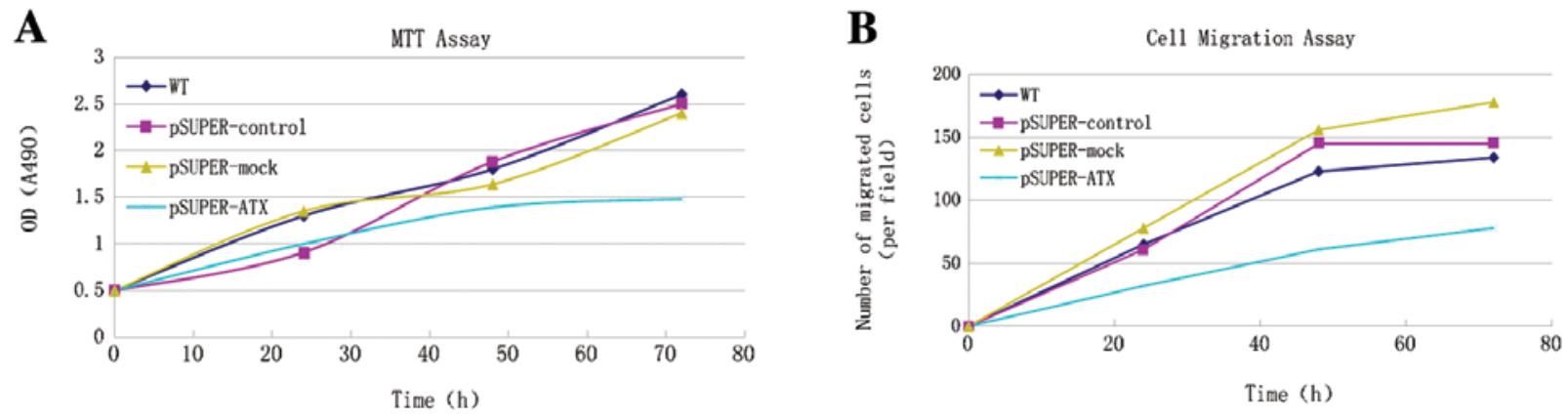

$\mathbf{C}$

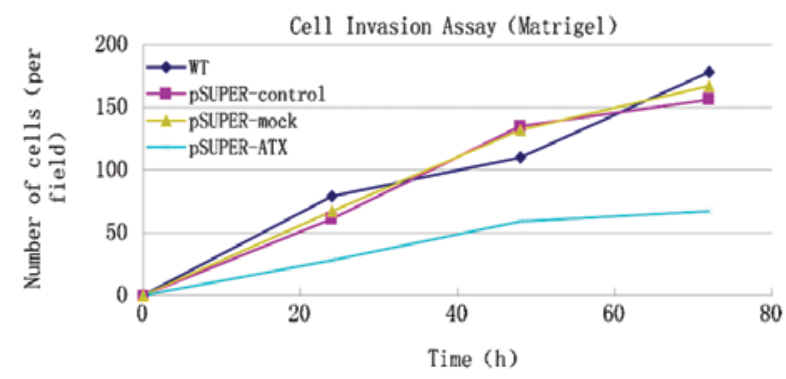

D

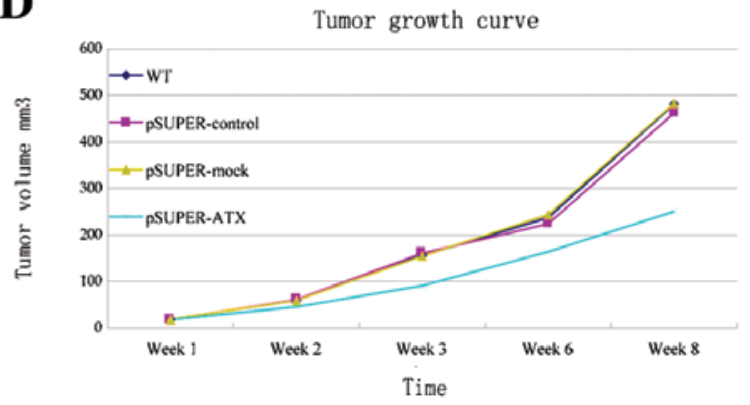

Figure 2. (A) Absorbance value of proliferating cells in the pSUPER-ATX group decreased, compared to the pSUPER-control and pSUPER-mock groups, but without significant differences $(P>0.05)$. (B) At 48 and $72 \mathrm{~h}$, the decrease in migrating cells in the pSUPER-ATX group was significant compared with the WT, pSUPER-control and pSUPER-mock groups. (C) At 48 and $72 \mathrm{~h}$, the decrease in invading cells in the pSUPER-ATX group was significant when compared with the WT, pSUPER-control and pSUPER-mock groups. (D) The growth curve of the xenograft tumors of the various mouse groups at different time periods.

for inhibiting the endogenous ATX expression. Therefore, the pSUPER-ATX 2 cell clone was selected for further in vitro and in vivo investigations.

Cell proliferation. MTT assay was performed to detect cell proliferation. As shown in Fig. 2A, the cell proliferative ability in the pSUPER-ATX group was constantly reduced $50 \mathrm{~h}$ after transfection, compared with the WT, pSUPER-control and pSUPER-mock groups. The number of living cells varied among the different groups which was propotional to the absorbance value. pSUPER-ATX had an inhibitory effect on AGS cell proliferation despite the statistical insignificance. 

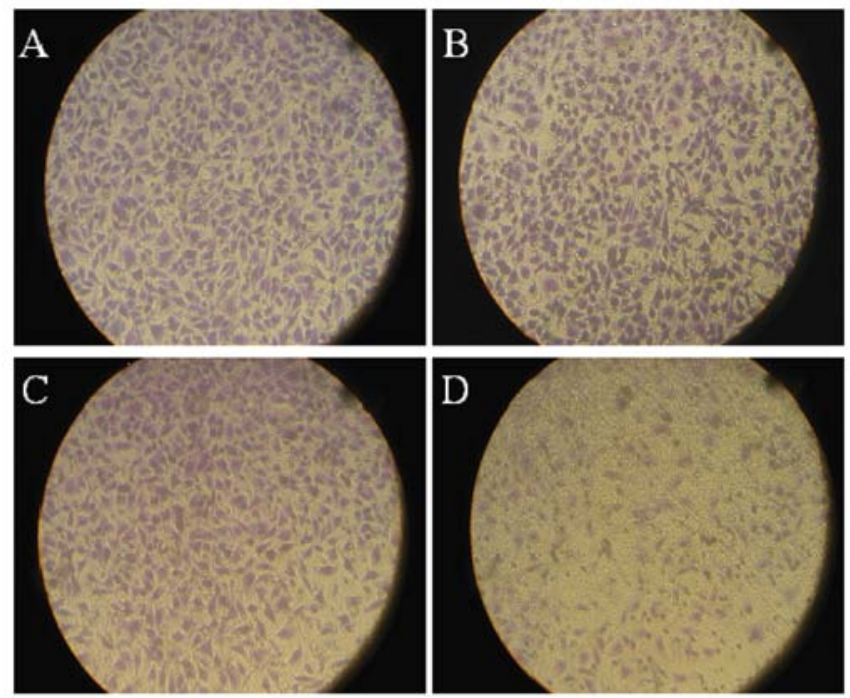

Figure 3. Cell invasion assay (Matrigel assay). Invading cells in the 4 groups: (A) wild-type, (B) pSUPER-control, (C) pSUPER-mock and (D) pSUPERATX.
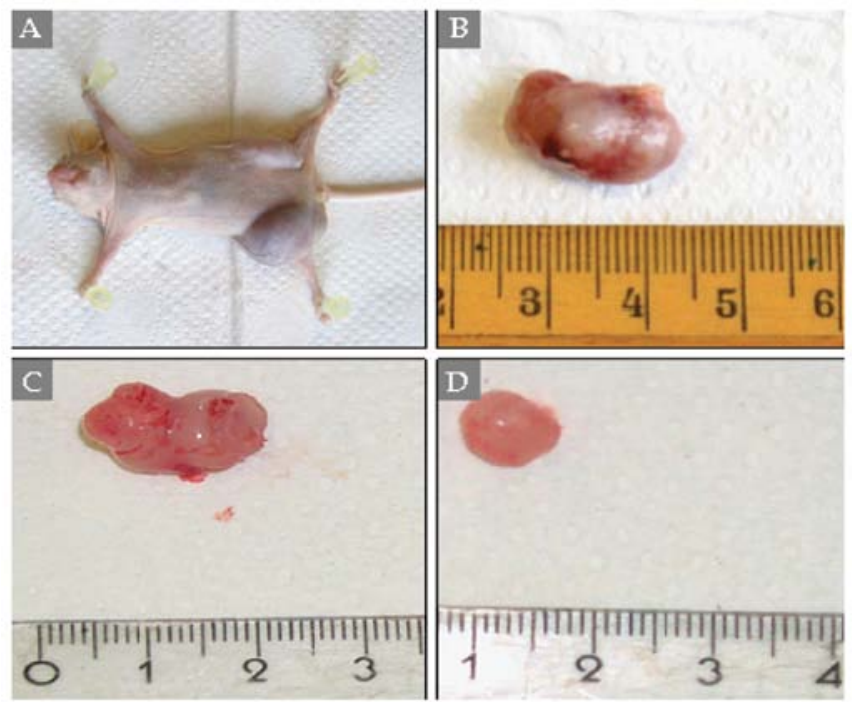

Figure 4. Specimens of the transfected cell line AGS xenograft tumors from the 8 th week after transplantation. (A) A nude mouse before being dissected. Tumors from the (B) WT, (C) pSUPER-mock and (D) pSUPER-ATX groups.

Cell migration and invasion. Cell migratory and invasive abilities were detected using Transwell and Matrigel assays (Fig. 3). From $20 \mathrm{~h}$ after transfection, migrating and invading cells in the pSUPER-ATX group decreased when compared with the WT, pSUPER-control and pSUPER-mock groups. From 48 to $72 \mathrm{~h}$ after transfection, the decrease in migrating and invading cells in the pSUPER-ATX group was significant but not in the WT, pSUPER-control and pSUPER-mock groups. As shown in Fig. 2B and C, the number of migrating cells decreased to $67.63 \pm 12.03 \%(\mathrm{t}=15.487, \mathrm{P}<0.01)$ and invading cells to $68.02 \pm 15.63 \%(t=9.417, \mathrm{P}<0.01)$. This indicated that pSUPER-ATX had an inhibitory effect on both migration and invasion.
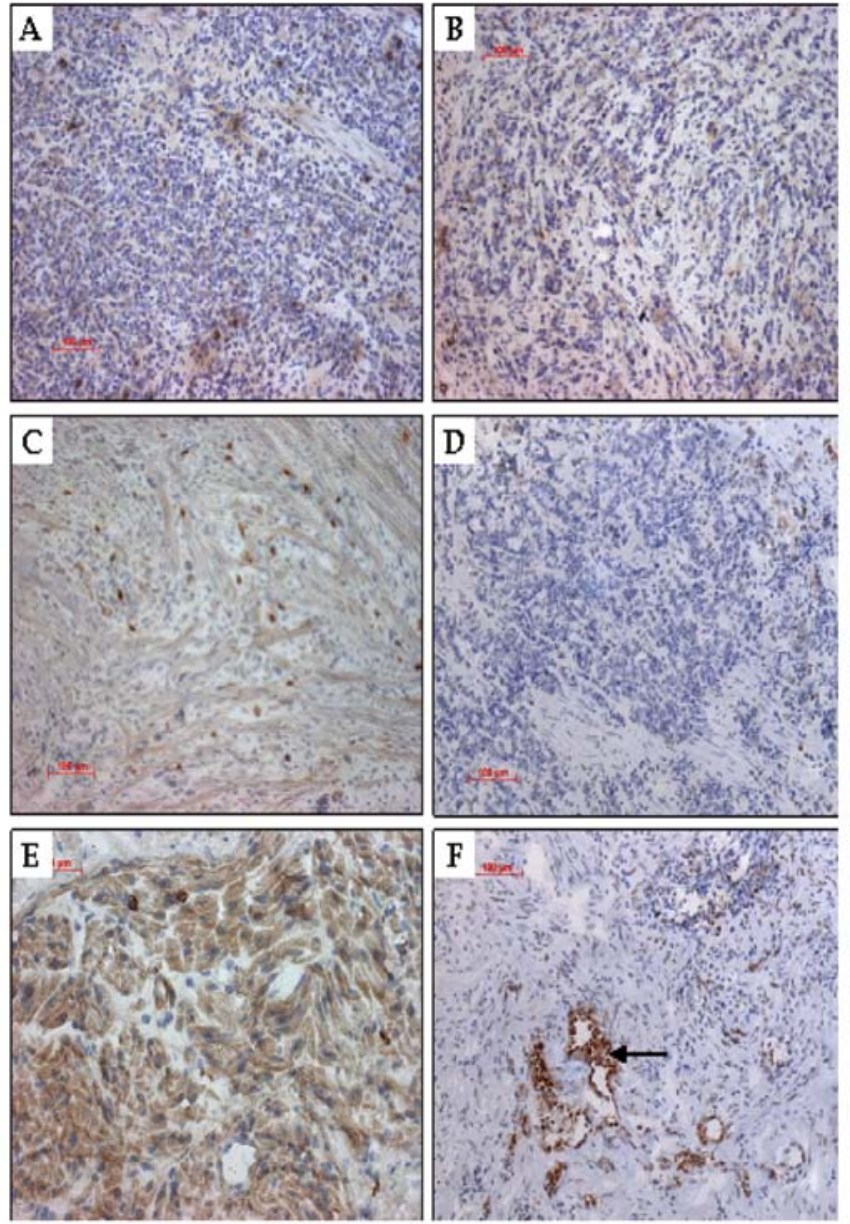

Figure 5. Immunohistochemical analysis of the expression of the ATX protein in xenograft tumors. ATX showed positive expression mostly in the (A) WT, (B) pSUPER-control and (C) pSUPER-mock groups. (D) ATX protein barely exhibited positive expression in the pSUPER-ATX group. Positive ATX expression in (E) xenograft tumor from the WT group at $\mathrm{X} 400$ amplification and in $(\mathrm{F})$ vascular endothelial (as shown by the arrow).

Western blot analysis. ATX protein expression in AGS cells was detected by western blot analysis. The internal control GAPDH was described as a homogeneous and positively expressed band, compared with the heterogeneously expressed band of ATX. ATX showed higher expression in the WT, pSUPER-control, pSUPER-mock, control and transfection groups, whereas ATX expression in the recombinant plasmid transfection group was decreased. Their differences were significant $(\mathrm{P}<0.05)$ (Fig. 1C). In comparion with the other groups, the endogenous protein expression of MMP-9, and particullarly, MMP-2 in the pSUPER-ATX group was noticeably reduced. The difference was significant $(\mathrm{P}<0.05)$ (Fig. 1D).

Growth of xenograft tumors in nude mice. Within an average of 8 days, a 3- to 4-mm diameter tumor developed at the subcutaneous injection sites of the right hind lateral leg of the nude mice with a $100 \%$ tumor formation rate (Fig. 4). Continuous observations during an 8-week period were carried out and a tumor growth curve was plotted (Fig. 2D). From the 16th day, 
Table I. Comparison of the xenograft tumor volume $\left(\mathrm{mm}^{3}\right)$ of each mouse group at different time periods.

\begin{tabular}{lccccc}
\hline Group & Week 1 & Week 2 & Week 3 & Week 6 & Week 8 \\
\hline WT & $18.1 \pm 0.3$ & $59.3 \pm 0.2$ & $155.1 \pm 4.1$ & $238.2 \pm 6.3$ & $477.1 \pm 8.4$ \\
pSUPER-control & $18.2 \pm 0.5$ & $60.2 \pm 1.1$ & $159.1 \pm 4.3$ & $221.3 \pm 5.1$ & $461.4 \pm 8.5$ \\
pSUPER-mock & $18.6 \pm 0.2$ & $58.9 \pm 0.9$ & $154.2 \pm 2.4$ & $241.9 \pm 5.8$ & $483.6 \pm 5.2$ \\
pSUPER-ATX & $17.9 \pm 0.2$ & $45.2 \pm 0.8$ & $90.7 \pm 3.5^{\mathrm{a}}$ & $163.5 \pm 6.2^{\mathrm{a}}$ & $248.2 \pm 6.9^{\mathrm{a}}$ \\
\hline
\end{tabular}

${ }^{a}$ Compared to the WT group, $\mathrm{P}<0.05$.

Table II. Parameters of the tumors at the 8th week following sacrifice of the nude mice.

\begin{tabular}{lccc}
\hline Group & BWC/g & $\begin{array}{c}\text { Tumor } \\
\text { weight }(\mathrm{g})\end{array}$ & $\begin{array}{c}\text { Inhibition } \\
\text { rate (\%) }\end{array}$ \\
\hline WT & 3.5 & $2.54 \pm 0.17$ & 0.0 \\
pSUPER-control & 2.9 & $2.21 \pm 0.21$ & 13.0 \\
pSUPER-mock & 3.1 & $2.36 \pm 0.15$ & 7.0 \\
pSUPER-ATX & 2.8 & $0.97 \pm 0.23$ & 61.8 \\
\hline
\end{tabular}

BWC, average body weight change. Inhibition rate $=(1-$ tumor weight of transfectant/tumor weight of WT) x $100 \%$.

the tumor volume between the pSUPER-ATX and the WT, pSUPER-control and pSUPER-mock groups showed a statistically significant difference $(\mathrm{P}<0.05)$; by the 8 th week, a tumor size of $477.1 \pm 8.4,461.4 \pm 8.5,483.6 \pm 5.2$ and $248.2 \pm 6.9 \mathrm{~mm}^{3}$ was, respectively, recorded in the WT, pSUPER-control, pSUPERmock and pSUPER-ATX groups (Table I). The tumor size of the pSUPER-ATX group was much smaller than the tumor sizes in the WT, pSUPER-control and pSUPER-mock groups $(\mathrm{P}<0.01)$, and no significant difference was detected between the negative and blank group $(\mathrm{P}>0.05)$. Nude mice in each group had a normal diet, good mental state, and exhibited no toxicity throughout the feeding process with inhibition of AGS proliferation by targeting shRNA. Until the sacrifice of nude mice in the 8th week, the average weight of the transplanted tumor in each group was $2.21 \pm 0.21,2.54 \pm 0.17,2.36 \pm 0.15$ and $0.97 \pm 0.23 \mathrm{~g}$. The inhibitory rates of tumor volume and weight in the interference pSUPER-ATX group were 52.1 and 52.3\%, respectively (Table II).

Immunohistochemical analysis of the expression of ATX protein in the xenograft tumors. $\mathrm{H} \& \mathrm{E}$ staining was used to demonstrate the inhibition of expression of the ATX protein by targeting shRNA. We observed malformed, large, deeply stained and eccentric nuclei in the transplanted tumor cells. Several cancer cells had multiple nuclei. There was no abnormal change observed in the hearts, livers, kidneys and spleens of the nude mice. A large number of brown-like cytoplasmic particles in the immunohistochemical slices of the blank and negative control groups was noted (Fig. 5).

\section{Discussion}

Invasion and metastasis of gastric cancer involve a complicated and continuous process involving multiple genetic regulations and factor interaction $(15,16)$. A number of different factors exert control of this invasion and metastasis process either by means of mono-regulation at different levels or mutual regulation (15). It involves structural and functional gene abnormalities associated with tumor invasion and metastasis. Dynamic cell movement is required in the process of transition from in situ cancer to invasive cancer, where cancer cells need to penetrate the basilar membrane and matrix gap (14). Euer et al used GeneChip technology to show that ATX was correlated with the regulation of high-level expression of more than 40 genes, which are intimately related to the highly metastatic characteristic of cells (17). The result of an in vitro test suggested that non-endogenous ATX-transfected ras-transformed NIH3T3 cells showed more enhanced invasive and metastatic potential (18). An in vitro cytological study confirmed that the ATX system was capable of mediating cell tumor migration (19). Furthermore an in vivo test directly identified ATX as an angiogenic factor, since ATX-transfected cells showed markedly enhanced angiogenic potential (20). Noh et al firstly used RNA interference to block ATX expression of breast cancer MDA435 cells and employed GeneChip technology to perform a spectral scan comparison of the parental cells and the gene expression of transfected cells. They found the most upregulated or downregulated cells had an intimate correlation with cellular metabolism, cytoskeleton fabric, transcriptional regulation and cell signal transduction. Therefore, ATX is thought to be a key regulator in phosphatidic acid metabolism and signal transduction. ATX also plays a role as a new molecular target for gene therapy against breast cancer (21).

In previous research, our group found that gastric cancer tissue samples in patients with distant and regional lymph node metastases showed extremely high ATX expression, compared with samples from patients without distant and lymph node metastases. The result indicated the ATX expression positively correlates with the metastatic potential of human gastric cancer cells. ATX in normal gastric tissue may contribute to regulation of cell growth, the exact mechanism of which, however, still needs to be further investigated. In the present study, RNA interference technology was employed to clone ATX via XhoI/ $B g I I$ into pSuper-neo-GFP with the H1 promoter, which was then transfected into the human gastric cancer AGS cell line characterized by low ATX expression and passaged in a stable 
process. The result from the western blot analysis indicated the AGS cells transduced with target shRNA were able to inhibit endogenous ATX mRNA and protein expression. After transfection, the migratory and invasive abilities of AGS cells with low ATX expression were significantly higher than these abilities in the wild-type cells. This indicates that AXT signal transduction pathways play an important role in the process of ATX-mediated cell invasion and migration. Interference with these pathways was thought effective in prevention of gastric cancer metastasis, which also allowed us to conclude that ATX could be a new molecular target for gastric cancer treatment. It has been speculated by our research group that ATX-mediated gastric cancer cell invasion and migration depends on RhoGTPase activation. The RhoGTPase family is a group of important proteins involved in a number of cell signaling transduction pathways, of which the members are key regulatory molecules that link membrane surface receptors to the organization of actin cytoskeleton. Changes in extracellular signal-induced actin cytoskeleton organization also cause a series of biological reactions, such as cell morphogenesis, chemotaxis and axon orientation, (22-24). Reymond et al comfirmed the Rho, Rac, $\mathrm{Cdc} 42$ and Ras promoted the regulation of signal transduction during regulation of the cytoskeleton, cell movement, cell proliferation and apoptosis (25). The results provide the basis for in vivo testing which is necessary for further research on the molecular mechanism of ATX in promoting gastric cancer invasion and metastasis. Our study indicates that the shRNA plasmid targeting autotoxin effectively downregulates the protein expression in vivo and inhibits the growth of AGS cells. Furthermore, by downregulating indirectly the protein levels of MMP-2 and MMP-9 of the MMP family, shRNA targeting ATX may reduce the invasive ability of gastric cancer cells.

The decomposition of the main components in basement membrane type IV and V collagen is carried out by MMP-2 and MMP-9. The basement membrane acts as a physiological barrier which needs to be overcome in the process of tumor infiltration and metastasis (26). Effective degradation of the extracellular matrix promotes tumor infiltration and metastasis. What is more, MMP-2 and MMP-9 are hypothesized to play a key role in the process of endothelial cell migration during angiogenesis. Additionally, synthesis and release of growth factors VEGF, bFGF and TGF- $\beta$ are promoted by MMP-2 and MMP-9 (27,28). Knockdown of ATX expression results in a decrease in MMP-2 and MMP-9 expression in gastric cancer, which can inhibit cancer infiltration and metastasis (29). Our study suggests that the ATX, Rho and MMP families are implicated to have pathways in gastric cancer signal transduction. Low expression of ATX, MMP2 and MMP9 contributes to the inhibition of cancer cell proliferation and metastasis, further affecting patient prognosis. The detailed signaling pathways and their mechanisms require further investigation. Our results provide new evidence concerning the molecular mechanisms of gastric cancer metastasis and may aid in developing clinical applications for gene therapy.

\section{Acknowledgements}

This study was funded by the Zhejiang Provincial Natural Science Foundation of China. We thank Mrs. Kathrin Hammje and Mr. Yuping Liu for their excellent technical and experimental assistance. We also deeply thank Ms. Ying Li for her helpful corrections and suggestions.

\section{References}

1. Stracke ML, Krutzsch HC, Unsworth EJ, et al: Identification, purification, and partial sequence analysis of autotaxin, a novel motility-stimulating protein. J Biol Chem 267: 2524-2529, 1992.

2. Umezu-Goto M, Kishi Y, Taira A, et al: Autotaxin has lysophospholipase D activity leading to tumor cell growth and motility by lysophosphatidic acid production. J Cell Biol 158: 227-233, 2002.

3. Tokumura A, Majima E, Kariya Y, et al: Identification of human plasma lysophospholipase D, a lysophosphatidic acid-producing enzyme, as autotaxin, a multifunctional phosphodiesterase. J Biol Chem 277: 39436-39442, 2002.

4. Kehlen A, Englert N, Seifert A, Klonisch T, Dralle H, Langner J and Hoang-Vu C: Expression, regulation and function of autotaxin in thyroid carcinomas. Int J Cancer 109: 833-838, 2004.

5. Saunders LP, Ouellette A, Bandle R, et al: Identification of small-molecule inhibitors of autotaxin that inhibit melanoma cell migration and invasion. Mol Cancer Ther 7: 3352-3362, 2008.

6. Debies MT and Welch DR: Genetic basis of human breast cancer metastasis. J Mammary Gland Biol Neoplasia 6: 441-451, 2001.

7. Yang SY, Lee J, Park CG, et al: Expression of autotaxin (NPP-2) is closely linked to invasiveness of breast cancer cells. Clin Exp Metastasis 19: 603-608, 2002.

8. Panupinthu N, Lee HY and Mills GB: Lysophosphatidic acid production and action: critical new players in breast cancer initiation and progression. Br J Cancer 102: 941-946, 2010.

9. Yang Y, Mou Lj, Liu N, et al: Autotaxin expression in non-small cell lung cancer. Am J Respir Cell Mol Biol 21: 216-222, 1999.

10. Stassar MJ, Devitt G, Brosius M, et al: Identification of human renal cell carcinoma associated genes by suppression subtractive hybridization. Br J Cancer 85: 1372-1382, 2001.

11. Deissler H, Blass-Kampmann S, Bruyneel E, et al: Neural cell surface differentiation antigen gp130(RB13-6) induces fibroblasts and glioma cells to express astroglial proteins and invasive properties. FASEB J 13: 657-666, 1999.

12. Zhang G, Zhao Z, Xu S, et al: Expression of autotoxin mRNA in human hepatocellular carcinoma. Chin Med J (Engl) 112: 330-332, 1999.

13. Chen Y, Wei X, Guo C, et al: Runx3 suppresses gastric cancer metastasis through inactivation of MMP9 by upregulation of TIMP-1. Int J Cancer 129: 1586-1598, 2011.

14. Gong M, Meng L, Jiang B, et al: p37 from mycoplasma hyorhinis promotes cancer cell invasiveness and metastasis through activation of MMP-2 and followed by phosphorylation of EGFR. Mol Cancer Ther 7: 530-537, 2008.

15. Schwartz GK: Invasion and metastases in gastric cancer: in vitro and in vivo models with clinical correlations. Semin Oncol 23: 316-324, 1996.

16. Yasui W, Oue N, Aung PP, Matsumura S, Shutoh M and Nakayama H: Molecular-pathological prognostic factors of gastric cancer: a review. Gastric Cancer 8: 86-94, 2005.

17. Euer N, Schwirzke M, Evtimova V, et al: Identification of genes associated with metastasis of mammary carcinoma in metastatic versus non-metastatic cell lines. Anticancer Res 22: 733-740, 2002.

18. Nam SW, Clair T, Campo CK, et al: Autotaxin (ATX), a potent tumor mitogen, augments invasive and metastatic potential of ras-transformed cells. Oncogene 19: 241-247, 2000.

19. Kishi Y, Okudaira S, Tanaka M, et al: Autotaxin is overexpressed in glioblastoma multiforme and contributes to cell motility of glioblastoma by converting lysophosphatidylcholine to lysophosphatidic acid. J Biol Chem 281: 17492-17500, 2006.

20. Tanaka M, Okudaira S, Kishi Y, et al: Autotaxin stabilizes blood vessels and is required for embryonic vasculature by producing lysophosphatidic acid. J Biol Chem 281: 25822-25830, 2006.

21. Noh JH, Ryu SY, Eun JW, et al: Identification of large-scale molecular changes of Autotaxin(ENPP2) knock-down by small interfering RNA in breast cancer cells. Mol Cell Biochem 288: 91-106, 2006

22. Li X and Lim B: RhoGTPases and their role in cancer. Oncol Res 13: 323-331, 2003. 
23. Bell CH, Aricescu AR, Jones EY and Siebold C: A dual binding mode for RhoGTPases in plexin signalling. PLoS Biol 9: e1001134, 2011.

24. Lazer G and Katzav S: Guanine nucleotide exchange factors for RhoGTPases: good therapeutic targets for cancer therapy? Cell Signal 23: 969-979, 2011.

25. Reymond N, Riou P and Ridley AJ: Rho GTPases and cancer cell transendothelial migration. Methods Mol Biol 827: 123-142, 2012.

26. Wiercinska E, Naber HP, Pardali E, van der Pluijm G, van Dam $\mathrm{H}$ and ten Dijke P: The TGF- $\beta /$ Smad pathway induces breast cancer cell invasion through the up-regulation of matrix metalloproteinase 2 and 9 in a spheroid invasion model system. Breast Cancer Res Treat 128: 657-666, 2011.
27. Kim ES, Kim MS and Moon A: Transforming growth factor (TGF)-beta in conjunction with H-ras activation promotes malignant progression of MCF10A breast epithelial cells. Cytokine 29: 84-91, 2005.

28. Chuang MJ, Sun KH, Tang SJ, et al: Tumor-derived tumor necrosis factor-alpha promotes progression and epithelialmesenchymal transition in renal cell carcinoma cells. Cancer Sci 99: 905-913, 2008.

29. Jiang WG, Raz A, Douglas-Jones A and Mansel RE: Expression of autocrine motility factor (AMF) and its receptor, AMFR, in human breast cancer. J Histochem Cytochem 54: 231-241, 2006. 